\title{
PREPARING OF THE CHAMELEON COATING BY THE ION JET DEPOSITION METHOD
}

\author{
Jakub Skocdopole $^{a, *}$, Lucrezia Aversa $^{b}$, Martin Golan $^{c}$, \\ Antonin Schenk ${ }^{d}$, Giacomo Baldi ${ }^{b}$, Irena KratochVilova ${ }^{c}$, \\ LadislaV KalvodA ${ }^{a}$, Petr NozaR ${ }^{b}$ \\ ${ }^{a}$ Department of Solid State Engineering, Faculty of Nuclear Sciences and Physical Engineering, Czech Technical \\ University in Prague, Prague, Czech Republic \\ ${ }^{b}$ Istituto Materiali per Elettronica e Magnetismo IMEM-CNR, Trento, Italy \\ ${ }^{c}$ Institute of Physics, CAS in Prague \\ d Department of Physical Electronics, Faculty of Nuclear Sciences and Physical Engineering, Czech Technical \\ University in Prague, Prague, Czech Republic \\ * corresponding author: jakub.skoc@gmail.com
}

\begin{abstract}
Preparation of chameleon coatings using an Ionized Jet Deposition (IJD) technique is reported in the present paper. IJD is a new flexible method for thin film deposition developed by Noivion, Srl. The chameleon coatings are thin films characterised by a distinct change of their tribological properties according to the external conditions. The deposited films of SiC and TiN materials were examined by the Raman spectroscopy, SEM and XPS. The results of the Raman spectroscopy have proved an amorphous structure of SiC films. The data from XPS on TiN films have shown that the films are heavily oxidized, but also prove that the films are composed of TiN and pure Ti. The SEM provided information about the size of grains and particles constituting the deposited films, which is important for tribological properties of the films. Deposition of the chameleon coating is very complex problem and IJD could be ideal method for preparation of this coating.
\end{abstract}

Keywords: Ion Jet Deposition, Chameleon coating, Raman spectroscopy, Scanning electron microscope, $\mathrm{X}$-ray photoelectron spectroscopy.

\section{INTRODUCTION}

Chameleon coating is a multilayer film, which changes its tribological attributes according to the external conditions. The main attributes interesting from a point of view of possible industrial applications are the capability of the layer to withstand high mechanical loads without a fracture, high wear resistance and low friction coefficient of the film.

Ionized Jet deposition is one of PVD (Physical Vapor Deposition) methods for preparation and growth of thin films. It is based on generation and application of dense pulsed electron beam which interacts with selected target material and produces a plume of the target material. The thin layer of target material is grown by condensing the plasma plume on the appropriate substrate.

The flexibility of the method stands in the set of independent parameters, variety of suitable materials for the deposition and in the possibility to use multiple IJD systems for the deposition of multilayers films. The independent parameters are distance between target and substrate, working gas, pressure during deposition, frequency of electron beam pulses and trigger voltage. All these parameters can be adjusted according to the desired deposition process outcome. The IJD technique permits to deposit both inorganic and organic materials on substrates which, again, could be of inorganic or organic nature because of low temperature of deposition plasma. It is also possible to deposit conducting and non-conducting/isolating materials and optically transparent materials. However, in order to deposit strongly isolating materials, it is necessary to add an auxiliary anode between IJD hollow cathode and target of the deposited material to electrically decouple the IJD system from the target.

From these characteristics it seems to be reasonable to assume that the IJD method could be ideal technique for the chameleon coatings preparation.

In this article we will focus on a part of the chameleon coatings, namely, hard metal carbides and nitrides. Such deposited hard films are examined by Raman spectroscopy, SEM and XPS and the results reported and discussed.

\section{THEORY}

\subsection{IONIZED JET DEPOSITION}

The IJD working tool - a pulsed energetic electron beam - is generated in the IJD source. The main part of the IJD source is a hollow flight-through anode/cathode. An electron discharge trigger is attached to the anode/cathode and serves for the controlled "firing" of the main discharge and for the generation of the dense electron pulse directed towards the tar- 


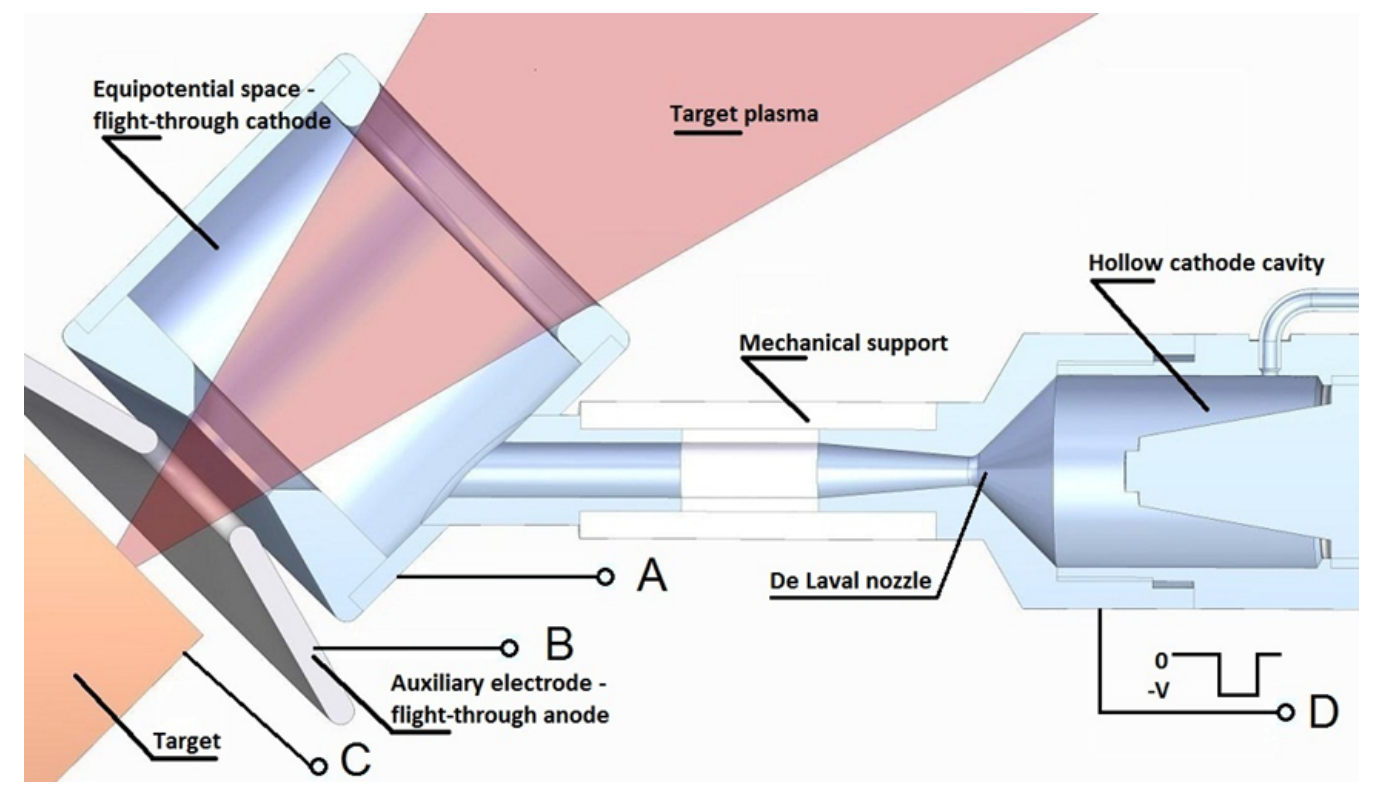

Figure 1. Example of an IJD source [8].

get. Usually (but not necessarily), either a small channel spark or a pseudospark switch, or an electron and plasma source is connected to the main anode/cathode and used as the trigger (see Figure 1). The main anode/cathode electrode has the form of a hollow cylinder with openings in both bases and oriented as follows: the base with the smaller opening towards the target and the base with the larger opening towards the substrate. The hollow cathode produces, owing to a Hollow cathode effect, a dense bunch of energetic electrons [7, that leave the hollow cathode towards the target in the final stage of the process. The electrons of the bunch hitting the target surface interact with the atoms of the target transferring to them their kinetic energy and creating a plume of energetic and highly ionized target plasma. The plasma plume which is predominantly expanding in the direction perpendicular to the target surface faces the hollow cathode, enters the smaller opening in the cylinder base facing the target, expands (flies) through the hollow cylinder and leaves the hollow cathode through the larger opening base towards the substrate (therefore "flight-through cathode").

\subsection{Chameleon COATing}

It is often necessary to use a combination of different materials within one thin film layer to optimize its selected properties. For example, it is possible to combine an amorphous matrix from DLC (diamond like carbon) with small grains of hard/ultra-hard material (such as tungsten carbide) within it. The grains should have the diameter in tens of $\mathrm{nm}$ and distance between them in $\mathrm{nm}$ for the best mechanical properties. The diamond like matrix provides the high hardness and low friction of deposited thin film, while the grains of carbide prevent the film from brittle fracturing and, if required, ensure the abrasive prop- erties of the film as e.g. in the case of cutting tools. Unfortunately, the low friction of this film occurs only in humid environment, because the DLC under the friction converts to graphite. The graphite does not have good wear resistance however it could be compensated by the grains of hard material. Graphite has low frictions coefficient only in humid environment. To provide low friction of the film in dry environment or in vacuum it is necessary to add another material e.g. MoS2 which has a hexagonal structure similar to the graphite but is excellent lubricant for dry environment or vacuum. To provide the low friction the MoS2 film has to present the hexagonal planes parallel to the friction force. Fortunately, the MoS2 material under friction has the tendency to orient its structure in such a way that it provides the lowest friction coefficient. Such ability allows substantial reduction of the system friction coefficient in dry environment just by adding the MoS2 material to the DLC matrix with carbide/nitrite particles incorporated. Film composition of MoS2/DLC/WC could be called chameleon because it adjusts its mechanical properties to the environment in which it is situated. In humid environment, the amorphous DLC provides low friction, the MoS2 and WC are scratched from the film surface in form of a debris. In dry environment the low friction is provided by MoS2, the graphitized DLC and WC are scratched from the surface [1] (Figure 2).

We assume that the IJD could be good choice for preparing such film. There are two options how the IJD could be used. First option uses two electron guns (IJDs) with two seperated targets (each for one IJD). Then the substrate must be placed in position where both plasma plumes overlap. The second option uses only one electron gun but needs rotary target holder. The rotary target holder provides opportunity 


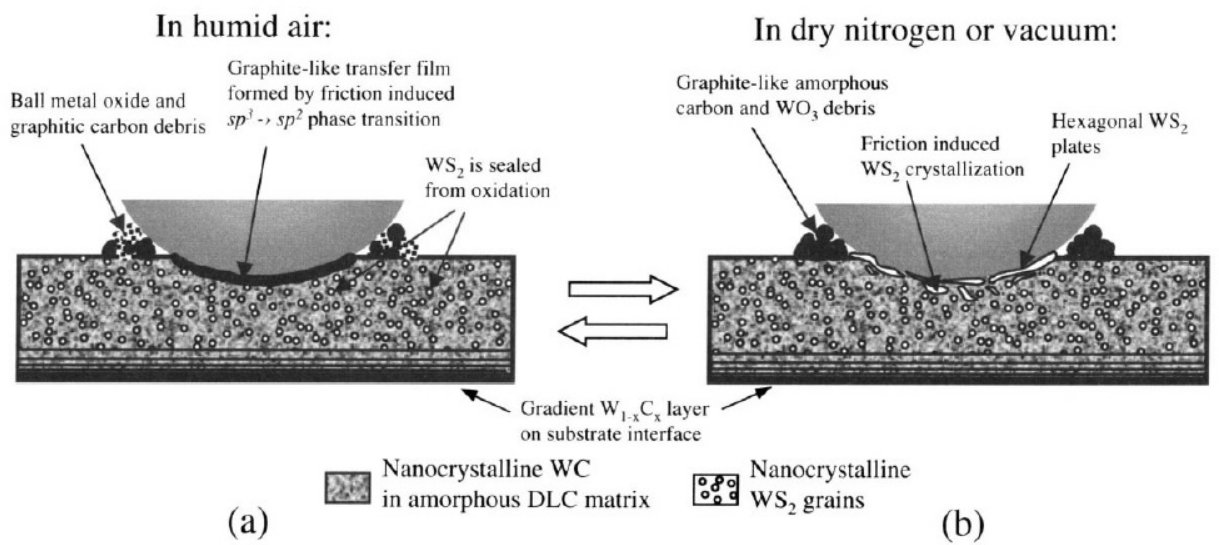

Figure 2. Principle of chameleon coating function [1].

\begin{tabular}{llllll}
\hline Name & Substrate & Film & Time $[\mathrm{min}]$ & Pressure $[\mathrm{mbar}]$ & Distance $[\mathrm{mm}]$ \\
\hline Kuba7 & Silicon wafer & TiN & 15 & 1.6 & 105 \\
\hline Kuba9 & Stainless-steel & TiN & 14 & 1.4 & 105 \\
\hline K2 & aluminium alloy & SiC & 30 & 0.6 & 180 \\
\hline
\end{tabular}

TABle 1. Deposition parameters.

of deposition from multiple targets only with one electron gun (IJD).

\section{EXPERIMENT}

Two types of hard material have been prepared trying to enhance the resistance of the deposited layer against fracturing, namely, the $\mathrm{SiC}$ deposited on aluminium alloy and TiN deposited on stainless steel and single crystalline silicon. All deposition events have been performed in Ar atmosphere. The deposition time, process gas (Ar) pressure during deposition and distance between the target and the substrate (see table 1) have been chosen as important process parameters to be adjusted for different deposited samples. The films have been analysed by Raman spectroscopy, SEM and XPS.

\section{Results}

The thickness of films was not directly measured, but it was deduced from the XRD results and from the values obtaind for similar films, which was deposited with the same deposition parameters. The roughly estimated thickness value obtaind in such way amounted several microns.

\subsection{RAMAN SPECTROSCOPY}

The measurement was performed at room temperature using an excitation laser source with the wavelength of $488 \mathrm{~nm}$. The exact positions of the peaks (bands) in the Raman spectrum were found by using a function findpeaks in matlab R2008b. The Raman spectrum of the sample K2 is shown in the Figure 3 .

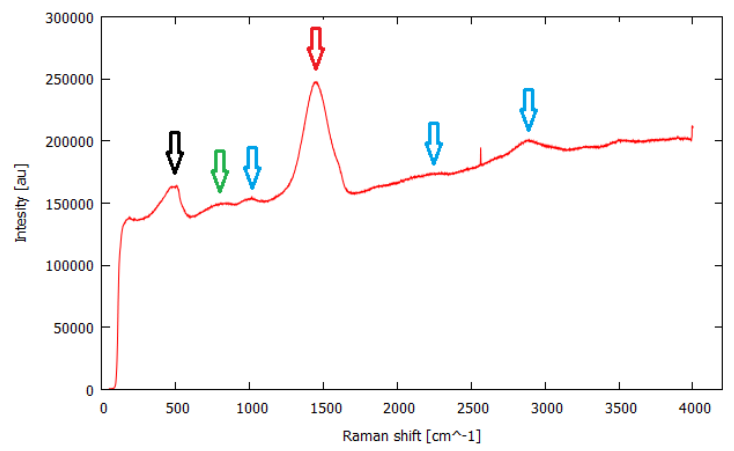

FiguRE 3. Raman spectrum of K2 sample.

The measured Raman band at $478 \mathrm{~cm}^{-1}$ (black arrow) is coresponding to $\mathrm{Si}-\mathrm{Si}$ bond in amorphous phase and $1448 \mathrm{~cm}^{-1}$ (red arrow) is coresponding to C-C amourphous bond. The Raman band at 829 $\mathrm{cm}^{-1}$ (green arrow) coresponds to $\mathrm{Si}-\mathrm{C}$ bond. The low intesity of Si-C peak is not due to the number of the bonds, but it follows from the generally small Raman transition probability. The bands at $1005 \mathrm{~cm}^{-1}, 2283$ $\mathrm{cm}^{-1}$ and $2889 \mathrm{~cm}^{-1}$ (blue arrows) link to the signals of Si-H and C-H bonds [2].

The Raman spectrum of the sample K7 and K9 is shown in Figure 4. According to [3], the peaks for TiN are found at 250;330-360 and $560 \mathrm{~cm}^{-1}$ and are marked by blue arrows. Observed peaks might be, perhaps, assigned to the presence of TiN in the deposited layer, however, the intensity of the peaks is too weak to provide a decisive prove for TiN compound existence. The peaks marked by black arrows correspond to monocrystalline silicon [6]. 


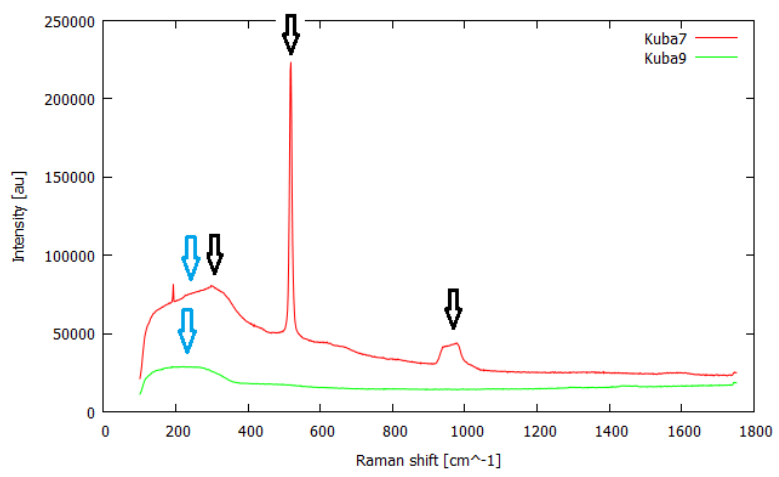

Figure 4. Raman spectra of samples K7 and K9.

\subsubsection{Discussion}

The Raman bands, for all samples, exhibit some shifts in their position, when compared with literature [2] [3]. This fact could be caused by some small variations of the stoichiometry of deposited films. For supposed application it is necessary to obtain well-defined grains of $\mathrm{SiC}$ or TiN. Unfortunately for this aim the thin films of $\mathrm{SiC}$ are amorphous. In order to be able to grow the polycrystalline SiC layers, it is necessary to heat the substrate during the deposition process, the treatment that was not performed during the present depositions. It is not possible to decide whether or not the thin films of TiN are really composed of pure TiN only. Or consist of some mixture of metallic Ti, TiN and titanium oxide. All these materials exhibit rather similar Raman spectra [4] and, taking in account the small intensity of the spectra obtained from present measurements it is impossible to conclude about the nature of grown material.

\subsection{XRD}

The glancing angle XRD analysis was performed on $\Theta / \Theta$ diffractometer X'Pert operating in BraggBrentano geometry with $\mathrm{X}$-Ray source $\lambda(\mathrm{CoK} \alpha$ ) $=0,179026 \mathrm{~nm}$.

The XRD patterns obtained with the sample K7 (Figure 5) show only amorphous halo. The amorphous halo is indicative of two facts, firstly that the film is thick enough that only the diffraction signal coming from the film is registered, and secondly that the structure of the films is amourphous in its nature. The XRD patterns obtained with sample K9 (Figure 6. show only presence of austenitic steel. The result indicates that the film is not thick enough to stop the diffraction signal from the substrate and that the structure of film is amorphous.

\subsection{SEM}

The SEM image of sample K2 (Figure 7) contains particles with size under $500 \mathrm{~nm}$. The film background is homogenous despite of fact that on its surface are particles. SiC is very hard material and was expected to consist of separated particles

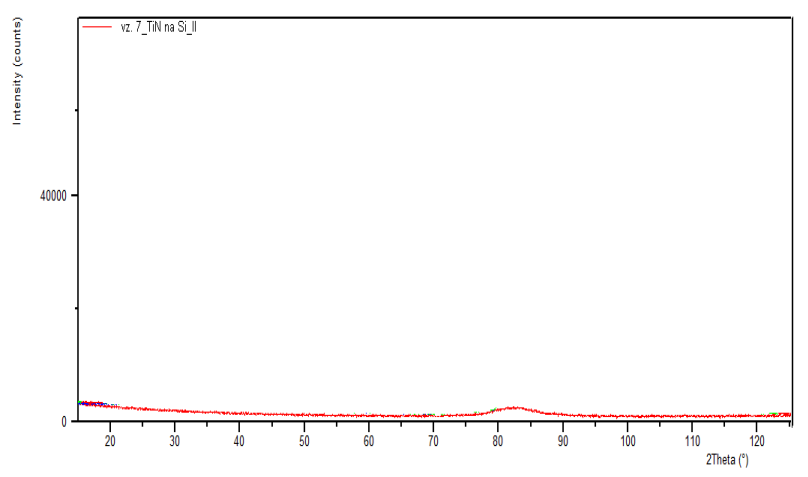

Figure 5. XRD patterns obtained with sample K7

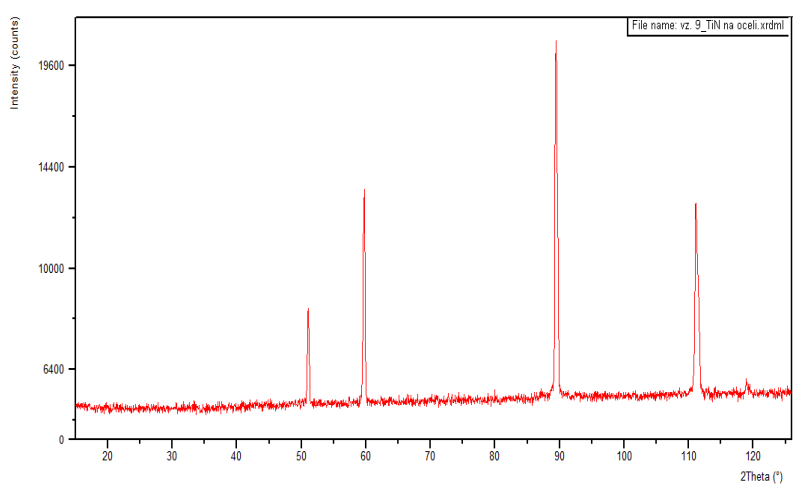

FIGURE 6. XRD patterns obtained with sample K9

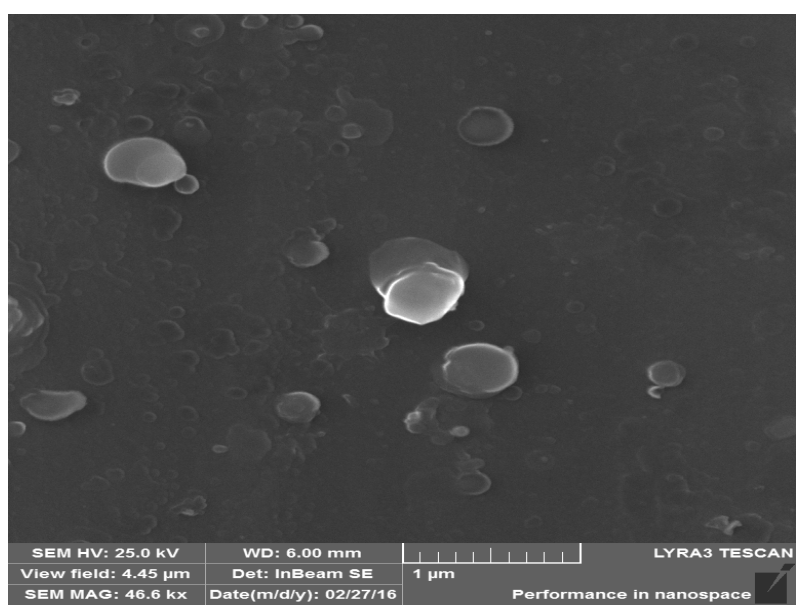

FiguRE 7. SEM micrograph of sample K2: SiC layer on aluminium alloy substrate.

The TiN was deposited on the stainless steel and monocrystalline silicon substrates. In SEM image of sample K7 (Figure 8) shows particles with size under $150 \mathrm{~nm}$. The film is very homogenous, in agreement with its amorphous nature confirmed by XRD.

The SEM image of sample K9 (Figure 9) shows particles under $250 \mathrm{~nm}$. The film is amourphous, as confirmed by XRD. The red lines indicate the differencies in the film structure due to existence of underlying grain boundaries present in the polycrystalline substrate. 


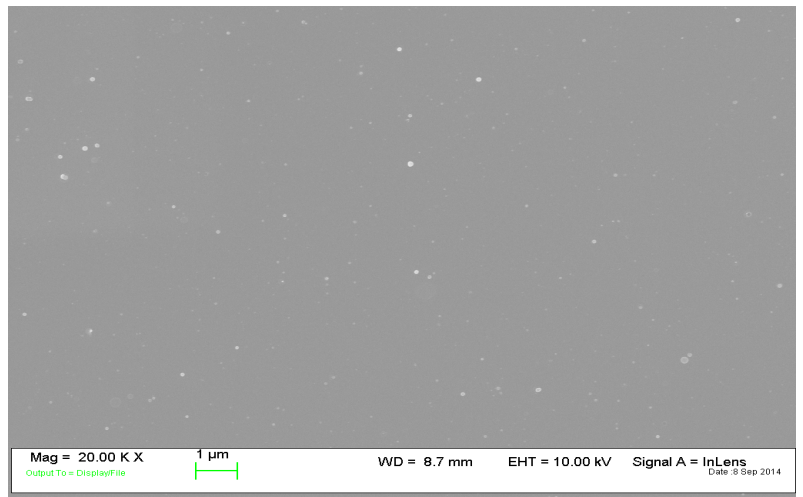

FIgURE 8. SEM micrograph of sample K7: TiN film on Si single crystal substrate.
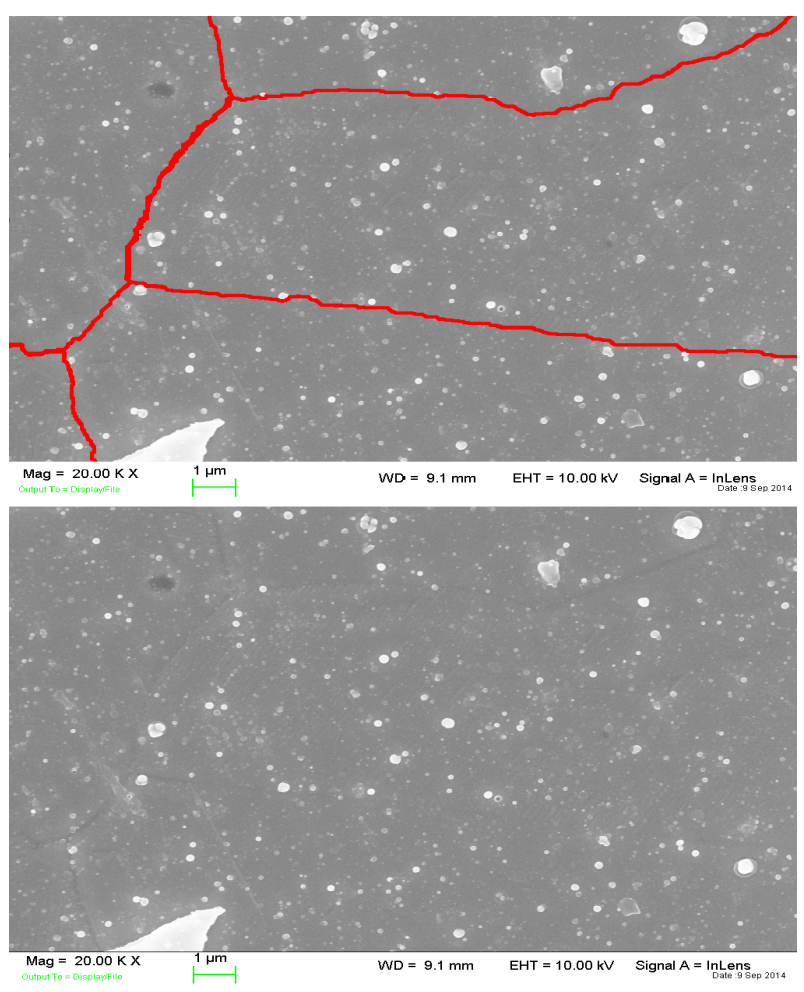

Figure 9. SEM micrograph of sample K9: TiN layer on stainless steel substrate.

\subsubsection{Discussion}

All samples exhibit some type of particles on their surface. The particles differ from each other mostly in their size. The size of particles is important for improvement of the mechanical properties. The particles should not be too large because in such case the cracks through the coating could propagate also through the particle and even not too small because the crack of the matrix may avoid the particle just by circling around it. The smallest particles are observed on the TiN film deposited on single crystalline silicon. The ideal size of the particles is found to be between tens and hundreds of nanometers [1].

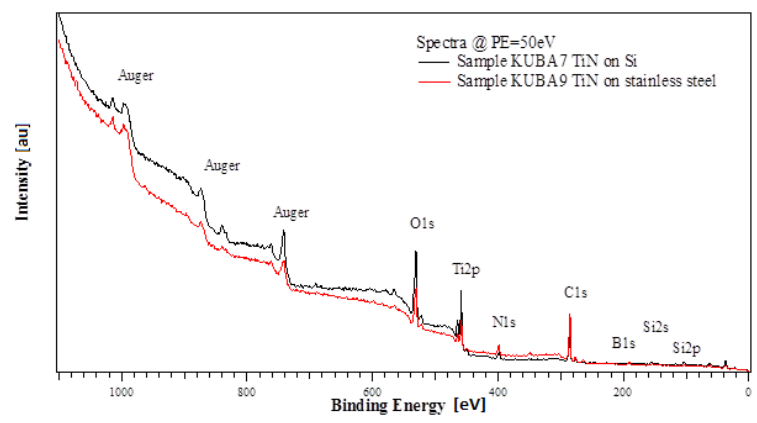

FiguRE 10. XPS spectra recorded with samples K7 and $\mathrm{K} 9 ; \mathrm{PE}=50 \mathrm{eV}$.

\subsection{X-RAY Photoelectron SPeCtroscopy}

The XPS analysis was performed using the X-ray source working at the MgKAlpha energy $1253.6 \mathrm{eV}$ and the WSW Analyzer that can be operated at different Pass Energy (PE), in order to collect spectra with different resolutions. In particular, the following settings were used: 1.) $\mathrm{PE}=50 \mathrm{eV}$ to acquiring the wide range spectra used in identification of the main chemical species on the surface 2.) $\mathrm{PE}=20 \mathrm{eV}$ to acquire single peak spectra with an intermediate resolution ideal to perform a quantitative analysis and 3.) $\mathrm{PE}=10 \mathrm{eV}$ to obtain single peak spectra at high resolution. The recorded wide range spectra are shown in Figure 10.

In the Ti-N samples, the main chemical species on the surfaces are those attended, i.e. titanium (Ti2p), nitrogen (N1s), oxygen (O1s), silicon (Si2p), carbon $(\mathrm{C} 1 \mathrm{~s})$ from contaminants and boron (B1s) from the PBN sample holder. The corresponding Auger electrons lines, that are always present in a typical XPS spectrum, are marked, too. The $\mathrm{O} 1 \mathrm{~s}$ and $\mathrm{C} 1 \mathrm{~s}$ peaks appear quite intense, in particular compared to the N1s peak that is expected to be stronger, even taking into account its low photoemission cross section: this suggests that the films could be strongly oxidized. Spectra at $\mathrm{PE}=20 \mathrm{eV}$ give more indication on oxidation processes together with the quantitative evaluation of surface atomic percentages, that are collected in Table 2

There is a significant amount of carbon that is present in $\mathrm{K}$ samples: the atomic percentages here reported take into account any contribution from the sample holder that has been properly subtracted from the $\mathrm{C} 1 \mathrm{~s}$ peak, thus the carbon percentage relies only on carbon inside or over the films.

The Ti2p peak is centered at a $\mathrm{BE}$ of $459 \mathrm{eV}$, the value that is typical of titanium in high oxidation state bonded to oxygen, and shows a long tail at lower BEs, usually related to lower valence states. The lineshape analysis (through Voigt functions, considering a Shirley background) put in evidence up to three contributions to the peak: the main one (light blue peak) is at higher $\mathrm{BE}$ and can be correlated to $\mathrm{TiO}_{2}$, the second one, centered at $457.5 \mathrm{eV}$, is out of the typical region of TiN (that is at even lower binding 


\begin{tabular}{llllll}
\hline Sample & \% Ti2p & \% N1s & \% O1s & \% C1s & \% Si2p \\
\hline KUBA7 & 13.2 & 3.3 & 38.5 & 38.2 & 6.8 \\
\hline KUBA9 & 7.2 & 1.2 & 24.3 & 67.3 & - \\
\hline
\end{tabular}

TABLE 2. Chemical composition obtained from XPS experiments for the K7 and K9 samples.

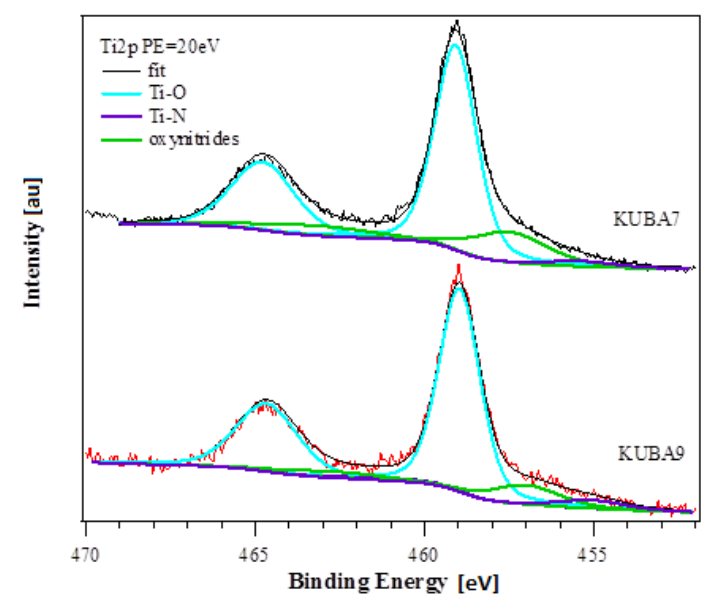

Figure 11. XPS spectrum close to Ti $2 p$ line for K7 and K9 samples.

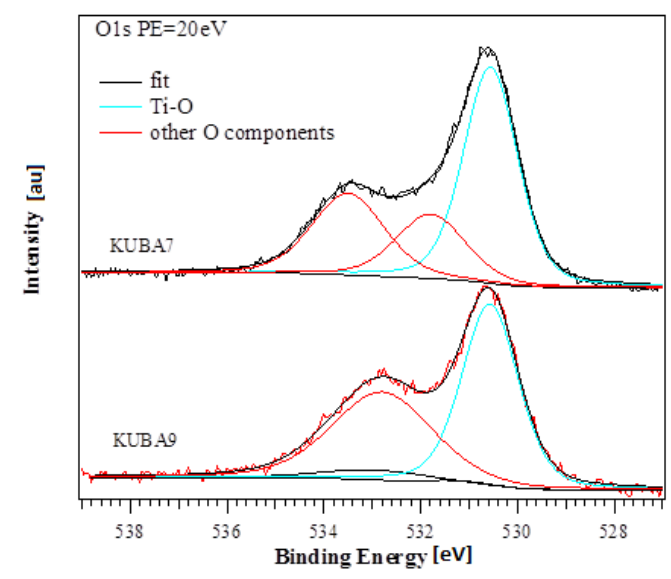

FiguRE 12. XPS spectrum courses close to O1s line observed for the K7 and K9 samples.

energy) and could be assigned to a mixed Ti-O-N phase (oxynitrides), while the third one at $455.4 \mathrm{eV}$ is the TiN contribution. Its intensity is low compared to the $\mathrm{TiO}_{2}$ phase, suggesting that the TiN layer is few nanometers deeper: the Ti2p escape length in $\mathrm{TiO}_{2}$ is $2.12 \mathrm{~nm}$, thus the signal from the nitride layer comes from about $6-8 \mathrm{~nm}$ below the surface. The O1s spectra for all samples show the presence of a main contribution at about $530-530.5 \mathrm{eV}$ and a less intense feature at higher binding energy. The lineshape analysis, however, reveals a third contribution at around $532 \mathrm{eV}$.

The most intense peak at low BE can be assigned to metal oxides, i.e. titanium oxides, while the peak at higher $\mathrm{BE}$ is typically assigned to $\mathrm{OH}$ groups at the surface. Taking into account the indications given

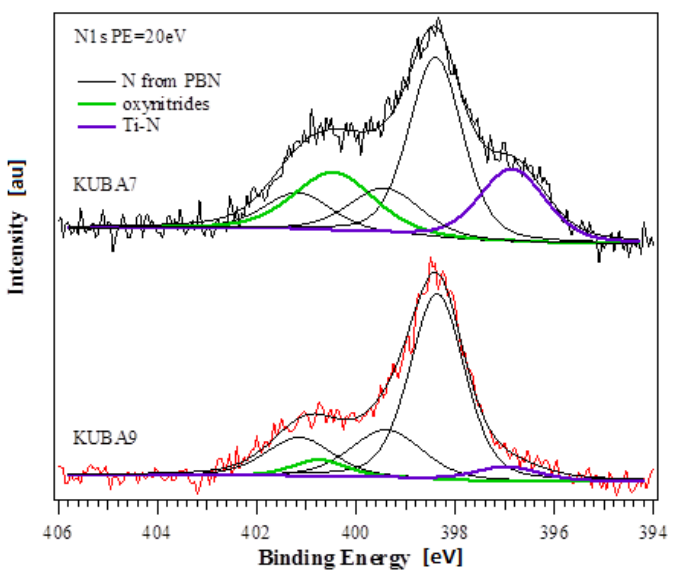

FigURE 13. XPS spectra close to N1s line observed for the K7 and K9 samples; experimental values (the noisy courses) and the fit curves are shown. For more details see text.

by Ti2p spectra, we can assume that this component together with the other $\mathrm{O}$ peak can be related to oxynitrides species. This assumption can be confirmed by looking at the N1s spectra (Figure 13).

Each nitrogen core level can be reproduced by the peak at low $\mathrm{BE}$ ( 396-397 eV, in blue and purple) that is assigned to the nitride layer, plus one or two components at higher $\mathrm{BE}$ that can be reasonably correlated to the oxynitrides already found in the O1s lineshape analysis.

\subsubsection{DisCussion}

The measurement has been performed before the $\mathrm{SiC}$ film has been prepared. Thus only TiN film is analyzed. It was expected that the measured data would originate mostly from the oxidation layer on the surface of the sample. This assumption was confirmed. The XPS does not prove unambiguously that the film is composed of TiN only. It seem reasonable to state that the XPS proves that Ti and N species are present in the film, however they could be combined in some mixture of $\mathrm{Ti}, \mathrm{TiN}$ and $\mathrm{Ti}-\mathrm{O}-\mathrm{N}$ (oxynitrides) phases.

\section{Conclusions}

Both films are composed of particles created during the deposition process, the feature which is necessary for their role in the chameleon coating. The deposition of particles from these materials were successful, however further research should be performed to ensure the reliable particle size control. The thickness of films is found to be in units of micrometres. Both 
TiN and SiC films are amorphous, as proven by Raman spectroscopy and XRD. Further research steps towards the chameleon layer preparation should be the deposition of $\mathrm{MoS}_{2}$ films and the incorporation of the $\mathrm{MoS}_{2}$ into DLC matrix and their characterisation.

\section{ACKNOWLEDGEMENTS}

The authors are indebted to Ing. Gianpiero Tedeschi, CEO, Noivion Srl, for providing the deposition technology for samples preparation and to the Institute of Materials for Electronics and Magnetism, CNR, Trento for its hospitality, especially to the Institute Director Dr. Salvatore Iannotta, and to the Trento Unit Head Dr. Roberto Verucchi. This work was supported by the Grant Agency of the Czech Technical University in Prague, grant No. SGS16/245/OHK4/3T/14.

\section{REFERENCES}

[1] A. A. Voevodin, J. S. Zabinski. Supertough wear-resistant coatings with 'chameleon' surface adaptation. Thin Solid Films 370: 223-231, 2000.

[2] Y. Inoue, S. Nakashima, A. Mitsuishi. Raman spectra of amorphous SiC. Solide State Communations 48(12): 1071-1075, 1983.

[3] W. Spengler, R. Kaiser. Raman scattering, superconductivity, and phonon density of states of stoichiometric and nonstoichimetric TiN. Physical Review B 17(3): 1095-1101, 1978.

[4] R. J. Nemanich, C. C. Tsai, G. A. N. Connell. Interference-Enhanced Raman Scattering of Very Thin Titanium and Titanium Oxide films. Physical Review Letters 44(4): 273-276, 1980.
[5] J.Skocdopole. Preparation of thin layers by IJD method and characterization of their physical-chemical properties.Prague. Bachelor thesis. Czech Technical University in Prague, Faculty of Nuclear Sciences and Physical Engineering, 2015.

[6] J.Skocdopole. Construction of system for deposition of thin films by IJD method and trial deposition of chosen materials..Prague. Research thesis. Czech Technical University in Prague, Faculty of Nuclear Sciences and Physical Engineering, 2016.

[7] S.O. Leathem,et al. Influence of the Injection Speeds on the Discharge Characteristics and Minimization of Delay Time in a Pseudospark Discharge. The Open Plasma Physics Journal 3: 20-27, 2010.

[8] P. Nozar. Ionized Jet Deposition Technique, a new class of thin film deposition equipment, Vacuum Symposium Coventry, 16 October 2014, the United Kingdom

[9] S.Tricot, et al. Dynamics of a pulsed-electron beam induced plasma: application to the growth of zinc oxide thin ïñ Ąlms Journal of Physics D 41(17): 10pp., 2008

[10] M.A. Raadu, J.J.Rasmussen. Dynamical aspects of electrostatic double layers Astrophysics and Space Science 144: 43-71, 1988

[11] Yu. D. Korolev, et al. Electron beams generated at different stages of the pseudospark discharge, The 27th International Conference on Phenomena in Ionized Gase, Eindhoven, 18-22 July 2005, the Netherlands 